\title{
Aptidão física relacionada à saúde de idosas da região do Vale do Sinos, RS: um estudo ex post-facto
}

\author{
Fitness related to health of elderly women of Vale do Sinos region, state of Rio Grande do Sul: an \\ ex post-facto research
}

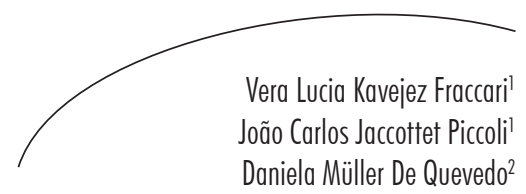

\section{Resumo}

Objetivo: $\mathrm{O}$ presente estudo verificou o nível de aptidão física relacionada à saúde de idosas de 60 a 70 anos, praticantes e não praticantes de atividades físicas de Sapiranga, RS, Brasil. Materiais e Métodos: Estudo ex post-facto que avaliou 40 idosas selecionadas por conveniência e distribuídas em dois grupos: praticantes de atividades físicas ( $\mathrm{n}=20$ ) e não praticantes de tais atividades $(\mathrm{n}=20)$. Foram avaliados: circunferência de cintura, razão cintura-quadril, índice de massa corporal (IMC), capacidade cardiorrespiratória, força muscular de membros superiores e inferiores e flexibilidade. A normalidade dos dados foi, inicialmente, verificada por meio do teste de Kolmogorov-Smirnov $(\alpha=$ 0,05). Para se traçar o perfil da amostra, fez-se uso da estatística descritiva, e para se verificar a diferença entre as médias dos dois grupos, utilizou-se o teste $t$ Student $(\alpha=$ $0,05)$ para amostras independentes, a partir do pacote estatístico SPSS, versão 16.0. Resultados: A idade média dos dois grupos avaliados foi de 64,6 $\pm 3,6$ anos e os resultados médios observados foram: IMC, 29,8 $\pm 5,7$; circunferência da cintura, $86,8 \pm 10,3 \mathrm{~cm}$; razão cintura-quadril, 0,86; teste de caminhada de 6 minutos, 409,1 $\pm 60,0 \mathrm{~m}$; frequência

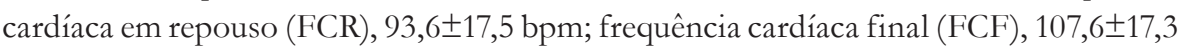
bpm; teste de flexão de cotovelo, 12,0 $\pm 3,7$ rep; teste de sentar e levantar da cadeira para

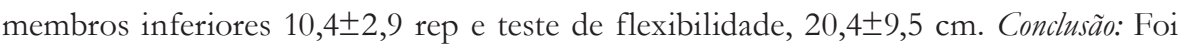
concluído que, nesta amostra, os resultados obtidos estão dentro do esperado para a faixa etária avaliada exceto na FCR e FCF.

\section{Abstract}

Objective: The purpose of study was to assess the level of fitness related to health in elderly women aged 60 to 70, physically active and sedentary, in the city of Sapiranga, state of Rio Grande do Sul, Brazil. Methods: It was an ex post-facto study that assessed 40 elderly women selected through a convenience sample and distributed into two groups: practicing physical activities $(n=20)$ and not practicing them $(n=20)$. It was assessed: waist circumference, Waist-hip ratio - WH-ratio, Body Mass Index-BMI,

Palavras chave: Idosos, Aptidão Física. Atividade física. Saúde. Sapiranga-RS/ Brasil.

\footnotetext{
1 Curso de Educação Física. Universidade Feevale. Novo Hamburgo, RS, Brasil.

2 Centro de Pesquisa e Planejamento. Universidade Feevale. Novo Hamburgo, RS, Brasil.

Key words: Elderly. Physical fitness. Physical activity, Health. Sapiranga-RS/Brasil. 
cardiorespiratory capacity, upper and lower limb strength, and flexibility. The data normal distribution was verified through the Kolmogorov-Smirnov test $(\alpha=0,05)$. The sample profile was determined through the descriptive Statistics and to test the difference between the two groups averages it was used the $t$ test for independent samples through the SPSS software, version 16.0. Results: The age average of the sample was $64.6 \pm 3,6$ years. The tests results average observed were: Body Mass Index, 29.8 \pm 5.7 ; waist circumference, $86.8 \pm 10.3 \mathrm{~cm}$; Waist-hip ratio, 0.86; 6-minute walk test, $409.1 \pm 60.0 \mathrm{~m}$; heart rate at rest (HRR), 93.6 $\pm 17.5 \mathrm{bpm}$; final heart rate

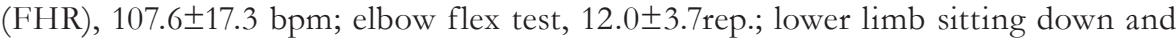
getting up from a chair, $10.4 \pm 2.9$ rep., and flexibility test, $20.4 \pm 9.5 \mathrm{~cm}$. Conclusion: The study conclusion was that in the studied sample the results were within the expected levels for the age group, except for HRR and FHR.

\section{INTRODUÇÃO}

$\mathrm{Na}$ Pesquisa Nacional por Amostra de Domicílios realizada em 2008, no Brasil, foi constatado que $48,9 \%$ e $54 \%$ dos idosos com 60 anos ou mais de idade ou a partir de 75 anos, respectivamente, sofriam de mais de uma doença crônica. Dentre elas, destacou-se a hipertensão $(53,3 \%)$ seguida pelas doenças de coluna ou costas $(35,1 \%)$, artrite ou reumatismo $(24,2 \%)$, diabetes $(16,1 \%)$ e outras doenças $(20,9 \%)$. Apenas $22,6 \%$ desses idosos alegaram não possuírem doenças. ${ }^{1}$

Quando estes dados são visualizados, pensase que muitas destas doenças crônicas são derivadas pelo avançar da idade que levam as funções musculares a uma redução gradativa e a sua interferência no desempenho das atividades da vida diária. ${ }^{2}$ Assim, envelhecer sem doença crônica é uma exceção, mas tê-la, não significa que o idoso deva se excluir socialmente. ${ }^{3}$ Para tal, manter a autonomia e a independência durante o processo de envelhecimento é uma meta a ser almejada, tanto, pelos indivíduos como profissionais e gestores públicos na área da saúde.

Ao se partir do pressuposto de que o idoso deva se manter ativo na sociedade, chega-se ao que se chama de envelhecimento ativo que tem por objetivo a melhoria da qualidade de vida por meio da otimização de oportunidades de saúde, participação e de segurança de idosos. A palavra "ativo" significa, então, estar fisicamente ativo, fazer parte da força de trabalho e participar de forma efetiva em questões sociais, econômicas, culturais, espirituais e civis. ${ }^{4}$
Neste artigo, considera-se parte deste conceito, isto é, a adoção de um estilo de vida saudável, por meio da prática de atividades físicas regulares e moderadas, pois tal prática poderá retardar o declínio funcional, além de diminuir o aparecimento de doenças crônicas no idoso, prevenir quedas e em consequência aumentar a longevidade e a sua qualidade de vida. ${ }^{3}$

A prática da atividade física é essencial em todas as fases da vida humana e é, ainda, mais importante na terceira idade, onde há uma perda da aptidão física e consequentemente da saúde. Uma boa manutenção da massa muscular e óssea contribui para uma maior autonomia de vida e na realização das atividades cotidianas do idoso. ${ }^{5}$

A aptidão física caracteriza-se, então, pela capacidade que um indivíduo possui para realizar atividades físicas, originada de fatores herdados, do estado de saúde, da alimentação e, principalmente, da prática regular de atividades físicas que pode ser mensurada subjetivamente pela quantidade de energia que uma pessoa possui para realizar coisas agradáveis na vida e estar preparada para enfrentar emergências sem fadiga excessiva. ${ }^{6,7}$

A aptidão física relacionada à saúde, uma das categorias da aptidão física que possibilita o idoso realizar as tarefas do dia a dia com o mínimo de fadiga e desconforto, é classificada em três componentes: aptidão cardiovascular, capacidade de continuar ou persistir em tarefas extenuantes que envolvam grandes grupos musculares e que requeiram 
muito oxigênio por longo período de tempo; aptidão musculoesquelética que é composta por uma combinação de três qualidades: força, resistência e flexibilidade e composição corporal que é a proporção de gordura e músculos e ossos responsáveis pelo tamanho do corpo. ${ }^{8}$

Para se verificar o nível de aptidão física relacionada à saúde de idosas de 60 a 70 anos, praticantes e não praticantes de exercícios físicos de Sapiranga,* RS, objetivo desta investigação, optou-se pela região do Vale do Sinos, berço da imigração alemã no Estado do Rio Grande do Sul.

\section{MATERIAIS E MÉTODOS}

A presente investigação, de característica ex post facto, investigou uma amostra composta por 40 idosas, com idades entre 60 e 70 anos, selecionadas por conveniência, e distribuídas em dois grupos: um formado por 20 praticantes de atividades físicas que realizavam alongamentos, ginástica, recreação, dança, caminhada e corrida orientada três vezes por semana por sessenta minutos no Programa de Ginástica para $3^{\mathrm{a}}$ idade de Sapiranga, RS e o outro, constituído, também, por 20 idosas, não praticantes de atividades físicas regulares que apenas executavam atividades domésticas.

A amostra foi finalmente estabelecida após as participantes terem sido esclarecidas e assinado o Termo de Consentimento Livre e Esclarecido TCLE. O estudo atendeu aos requisitos éticos da pesquisa, tendo sido aprovado pelo Comitê de Ética em Pesquisa da Universidade Feevale sob $n^{\circ}$. 4.09.03.09.1543.

Para a coleta das medidas da massa corporal e estatura, foram utilizadas uma balança da marca Ga Ma Italy professional e fita antropométrica em aço Sanny, 2 m (TR - 4011), American

\footnotetext{
* Cidade gaúcha localizada na Região do Vale do Rio dos Sinos, possui uma área territorial de $138,315 \mathrm{~km}^{2}$ e uma população constituída por 74.985 habitantes e uma densidade demográfica de 542,13 habitantes por $\mathrm{km} 2$. A população masculina é de 36.989 $(49,3 \%)$ e a feminina, 37.996 (50,6\%). Desta população, 96,4\% encontram-se na zona urbana e $3,6 \%$ na rural. Dentre a população total, $2.796(3,7 \%)$ são idosos do sexo masculino, acima de 60 anos e $3.802(10 \%)$, do sexo feminino. ${ }^{9}$
}

Medical do Brasil Ltda., com tolerância de $\pm 0,10$ $\mathrm{mm}$ em $1 \mathrm{~m}$. As avaliadas foram orientadas a se manterem descalças sobre a balança, com os braços soltos ao longo do corpo e com roupas leves. Para a medição da estatura, as participantes permaneceram encostadas em uma parede, com o olhar fixo no horizontal, possibilitando a posição da linha do queixo paralela ao solo.

O Índice de Massa Corporal - IMC foi calculado e utilizaram-se os pontos de corte propostos por Lipschitz: $:^{10}$ desnutrição: $<22,0 \mathrm{~kg} /$ $\mathrm{m}^{2}$, eutrofia: de 22,0 a $27,0 \mathrm{~kg} / \mathrm{m}^{2}$ e obesidade: $>27,0 \mathrm{~kg} / \mathrm{m}^{2}$.

Para a obtenção da medida da circunferência da cintura (CC) foi utilizada uma fita antropométrica, ficando as idosas em posição ortostática, de perfil para o avaliador, abdômen relaxado, braços erguidos lateralmente à altura dos ombros. A circunferência da cintura foi medida na cintura natural, ou seja, entre as costelas inferiores e as cristas ilíacas. A leitura foi feita no momento da expiração, e realizouse no milímetro mais próximo. A circunferência do quadril (CQ) foi verificada no nível da sínfise púbica com a fita circundando o quadril na parte mais saliente entre a cintura e a coxa e com o indivíduo usando roupas finas.

O acúmulo de gordura na cintura ou obesidade abdominal foi classificado como risco elevado e muito elevado para mulheres uma CC $\geq 80,0$ e $\geq 88,0 \mathrm{~cm}$, respectivamente. Valores abaixo de $80,0 \mathrm{~cm}$ foram classificados como normais. ${ }^{11} \mathrm{~A}$ razão cintura-quadril (RCQ), também, aferida, relacionada ao risco cardiovascular era aquela que fosse superior a 0,85 para mulheres; um valor inferior a 0,85 classificava-se como padrão normal. ${ }^{11}$

O teste de 6 minutos foi utilizado para verificar a capacidade cardiorrespiratória (resistência aeróbia) tendo as participantes, de forma contínua, percorrido a maior distância possível no tempo destinado ao teste, num percurso retangular plano de 45,7m (18,2 $\mathrm{m}$ x 4,5 $\mathrm{m})$ cujos ângulos internos da distância medida estavam demarcados por cones e as linhas de 4,5 $\mathrm{m}$, traçadas com giz. ${ }^{12}$ 
A frequência cardíaca (FC) foi medida através de frequencímetros de marca Polar ao final de cada minuto do teste quando se solicitava a participante o valor da FC indicada em seu monitor de frequência cardíaca. A exceção a essa regra se dava quando, por algum motivo, não era possível a sua leitura como, no caso de idosas com mamas avantajadas, perda de sinal do aparelho e roupas inadequadas, dentre outros. Nesse caso, verificou-se apenas a medida de $\mathrm{FC}$ final $\left(6^{\circ}\right.$ minuto) em artéria radial durante 15 segundos, fazendo a projeção para 1 minuto, seguindo protocolo sugerido por Pollock \& Wilmore..$^{13}$ Para a realização deste teste, foram, também, utilizados os seguintes materiais: trena para medir, giz para marcar os metros e traçá-los (para delimitar o retângulo), dois tonéis e duas cadeiras.

Durante o teste, eram avaliadas quatro idosas por vez, como também, utilizavam-se palavras encorajadoras, como "Você está indo bem, parabéns, continue!”. A avaliada era, também, responsável pela contagem do número de voltas percorridas. Ao final do teste cujo local fora demarcado previamente, anotava-se o percurso em metros e a participante era liberada para um breve repouso ativo, antes de se sentar.

Para o teste de força muscular de membros superiores, os voluntários permaneceram sentados em uma cadeira com as costas retas tocando o encosto e os pés apoiados no chão e tomou-se o cuidado de posicionar o lado dominante mais próximo à extremidade da cadeira. Ao comando verbal de iniciar dado pelo avaliador, o idoso flexionava o cotovelo, girando a palma da mão para cima (supinação). Orientouse para que todo o ângulo de movimento fosse cumprido, assim como o retorno à posição inicial com o cotovelo completamente estendido.

O avaliador fez uma rápida demonstração do teste e as idosas o experimentaram antes de seu início, para após, executarem o máximo de repetições em 30 segundos. Apenas contaram-se as repetições corretas e completas até o final dos 30 segundos; no caso de a avaliada ter realizado mais da metade do movimento ao final dos 30 segundos, este também era contabilizado. ${ }^{12}$
Em relação ao teste de força de membros inferiores, cada participante foi orientada a se sentar no meio da cadeira, mantendo suas costas retas, pés apoiados no chão e braços posicionados em forma de "x", apoiados na frente do tórax. Após a explicação, demonstração pelo avaliador e tentativa de familiarização com o movimento, cada idosa se levantava e sentava na cadeira completamente durante 30 segundos, procurando alcançar o máximo de repetições nesse tempo. Foram pontuados os movimentos completos realizados durante o tempo previsto para o teste e aqueles quase finalizados.

Para se medir a flexibilidade utilizou-se o teste sentar e alcançar (banco de Wells) que consistia em uma caixa de madeira medindo $30,5 \mathrm{~cm}$ x $30,5 \mathrm{~cm} \times 30,5 \mathrm{~cm}$, com um prolongamento de $23 \mathrm{~cm}$ para o apoio dos membros superiores das participantes. Sobre a face superior da caixa e do prolongamento foi afixada uma escala métrica de $53 \mathrm{~cm}$ que permitia determinar o alcance da participante cuja planta do pé encontrava-se apoiada na face superior da caixa e coincidia com o $23^{\circ} \mathrm{cm}$ da fita métrica.

Com os braços estendidos à frente, tendo uma das mãos sobreposta à outra, as idosas eram orientadas a flexionar o tronco e progredir lentamente para frente, deslizando as mãos sobre a fita métrica até atingir seu ponto individual máximo. ${ }^{14}$ A medida era anotada durante a fase de expiração, no ponto máximo em que o avaliado sustentava a posição por pelo menos 2 segundos. Três tentativas foram realizadas e anotadas em centímetros o melhor resultado.

Os dados foram submetidos, inicialmente, ao teste de normalidade de Kolmogorov-Smirnov $(\alpha=0,05)$ (Tabela 1) para verificar se as variáveis possuíam distribuição normal. Através do teste foi possível identificar que as variáveis em estudo possuíam distribuição normal, e neste caso para a inferência estatística, utilizaram-se testes paramétricos de comparação de médias.

Para se traçar o perfil da amostra fez-se uso da estatística descritiva (média e desvio-padrão) e para se verificar a existência de diferença entre as médias nos resultados dos testes dos dois grupos 
estudados utilizou-se o teste $t$ Student $(\alpha=0,05)$ para amostra independentes, a partir do pacote estatístico SPSS, versão 16.0.

\section{RESULTADOS}

A amostra do presente estudo, de uma forma geral, foi caracterizada por idosas com idade média de 64,6 \pm 3,6 anos; índice de massa corporal de 29,8 $\pm 5,7$, portanto, obesa, segundo os pontos de corte propostos por Lipschitz; ${ }^{10}$ com circunferência média de cintura de $86,8 \pm$ $10,3 \mathrm{~cm}$ e razão cintura-quadril de 0,86 , o grupo avaliado encontra-se em situação de risco para o desenvolvimento de doenças coronarianas (Tabela 1).

Tabela 1 - Distribuição total das médias, desvios-padrão (DP) e teste de normalidade de KolmogorovSmirnov das variáveis analisadas ( $\mathrm{n}=40)$. Sarapiranga, RS, 2010.

\begin{tabular}{lrrrr}
\hline \multicolumn{1}{c}{ Variáveis } & Média & DP & KS & p-value \\
\hline Idade (anos) & 64,6 & 3,6 & 0,119 & $>0,150$ \\
Massa corporal (Kg) & 69,8 & 12,6 & 0,136 & 0,063 \\
Estatura (m) & 1,5 & 0,07 & 0,109 & $>0,150$ \\
IMC (índice) & 29,8 & 5,7 & 0,120 & 0,148 \\
Circunferência do quadril (cm) & 100,6 & 8,4 & 0,071 & $>0,150$ \\
Circunferência da cintura (cm) & 86,8 & 10,3 & 0,092 & $>0,150$ \\
Frequência cardíaca em repouso (bpm) & 93,6 & 17,5 & 0,099 & $>0,150$ \\
Frequência cardíaca final (bpm) & 107,6 & 17,4 & 0,071 & $>0,150$ \\
Teste de 6 min.(m) & 409,1 & 60,0 & 0,084 & $>0,150$ \\
Força muscular de membros superiores (rep) & 12,0 & 3,7 & 0,088 & $>0,150$ \\
Força muscular de membros inferiores (rep) & 10,4 & 2,9 & 0,054 & $>0,150$ \\
Flexibilidade (cm) & 20,4 & 9,5 & 0,087 & $>0,150$ \\
\hline
\end{tabular}

As idosas apresentaram uma frequência cardíaca em repouso e final média de 93,6 \pm 17,5 e $107,6 \pm 17,4 \mathrm{bpm}$, respectivamente e os seguintes resultados médios nos testes aplicados: teste de seis minutos, 409,1 $\pm 60,0 \mathrm{~m}$; força muscular de membros superiores e inferiores, $12,0 \pm 3,7$ e $10,4 \pm 2,9$ repetições respectivamente e de flexibilidade, 20,4 \pm 9,5 cm (Tabela 1). 
Tabela 2 - Distribuição das médias, desvios-padrão (DP), teste " $t$ " e valor de $p$ das variáveis analisadas, separadas por grupo de participantes $(\mathrm{n}=40)$. Sarapiranga, RS, 2010.

\begin{tabular}{|c|c|c|c|c|c|c|}
\hline \multirow{2}{*}{ Variáveis } & \multicolumn{2}{|c|}{ Praticantes $(\mathrm{n}=20)$} & \multicolumn{2}{|c|}{ Não praticantes $(\mathrm{n}=20)$} & \multirow[t]{2}{*}{$\mathrm{t}$} & \multirow[t]{2}{*}{$p$} \\
\hline & $\bar{X}$ & $\mathrm{dp}$ & $\bar{X}$ & $d p$ & & \\
\hline Massa corporal $(\mathrm{kg})$ & 64,4 & 12,0 & 75,2 & 11,0 & $-2,969$ & $0,005^{* *}$ \\
\hline Estatura (m) & 1,5 & 0,05 & 1,5 & 0,08 & 0,878 & 0,386 \\
\hline IMC (índice) & 27,1 & 4,7 & 32,6 & 5,3 & $-3,488$ & $0,001^{* *}$ \\
\hline Circunferência do quadril (cm) & 97,0 & 6,6 & 104,1 & 8,6 & $-2,914$ & $0,006^{* *}$ \\
\hline Circunferência da cintura $(\mathrm{cm})$ & 80,9 & 8,4 & 92,7 & 8,6 & $-4,362$ & $0,000^{* *}$ \\
\hline $\begin{array}{l}\text { Frequência cardíaca em repouso } \\
\text { (bpm) }\end{array}$ & 99,3 & 19,8 & 88,0 & 13,1 & 2,133 & 0,039 \\
\hline Frequência cardíaca final (bpm) & 111,6 & 19,8 & 103,5 & 13,9 & 1,495 & 0,143 \\
\hline Teste de 6 min.(m) & 434,9 & 52,4 & 383,3 & 57,0 & 2,976 & $0,005^{* *}$ \\
\hline $\begin{array}{l}\text { Força muscular de membros } \\
\text { superiores (rep) }\end{array}$ & 13,8 & 3,9 & 10,2 & 2,4 & 3,482 & $0,001 * *$ \\
\hline $\begin{array}{l}\text { Força muscular de membros } \\
\text { inferiores (rep) }\end{array}$ & 12,2 & 2,0 & 8,6 & 2,5 & 4,975 & $0,000^{* *}$ \\
\hline Flexibilidade $(\mathrm{cm})$ & 22,3 & 9,9 & 18,4 & 9,0 & 1,310 & 0,198 \\
\hline
\end{tabular}

A tabela 2 apresenta maior detalhamento da amostra, separando-a por grupo, praticante e não praticante de atividade física. Quando se classificou a amostra do estudo como obesa, observa-se que o grupo de não praticantes de atividades físicas apresentou um IMC superior $(32,6)$ ao que as praticavam $(27,1)(p \leq 0,01)$, este, situando-se na linha divisória entre a eutrofia e obesidade. Tal fato, também, foi observado nas medidas de circunferência da cintura $(92,7$ / 80,9 $\mathrm{cm})$ e do quadril $(104,1 / 97,0 \mathrm{~cm})(p \leq 0,01)$ dos respectivos grupos.

Quando se verifica a relação cintura-quadril - RCQ, tomando-se como referência os pontos de corte da Organização Mundial da Saúde para mulheres: favorável $\leq 0,85$ e desfavorável $>85,{ }^{11}$ constata-se que o grupo ativo apresentou um RCQ de 0,83 e o grupo não ativo, 0,89, o primeiro, dentro dos padrões da normalidade e o último, apresentando risco para doenças cardiovasculares.
Em relação à média das frequências cardíacas em repouso e final (Tabela 2), observa-se que as praticantes de atividades físicas apresentaram uma média superior (99,3 e 111,6 bpm), em relação às não praticantes de atividades físicas regulares (88,0 e 103,5bpm). Entretanto, não se verificou diferença estatisticamente significativa entre os dois grupos, nesta variável ( $\mathrm{p} \geq 0,05)$.

Na tabela 2, verifica-se, através do teste $t$ Student que existe diferença estatisticamente significativa $(\mathrm{p} \leq 0,01)$ entre os grupos praticantes e não praticantes de atividade física em relação à média de distância percorrida em metros. Observa-se que o grupo ativo percorreu uma distância média superior (434,9 m) do que o não ativo $(383,3 \mathrm{~m})$.

No teste de flexão de força muscular de membros superiores, mais especificamente, teste de flexão de cotovelo, observa-se que o grupo que participava do Programa de Ginástica para $3^{a}$ 
idade apresentou um valor médio de flexões (13,8 $\mathrm{cm})$ superior ao que dele não participava $(10,2 \mathrm{~cm})$ $(p \leq 0,01)$. No que se refere ao teste de levantar da cadeira, de força muscular de membros inferiores, os resultados foram favoráveis, também, ao primeiro grupo $(\mathrm{p} \leq 0,01)$ (Tabela 2$)$.

Analisando-se a tabela 2, constata-se que o grupo não ativo apresentou uma flexibilidade média inferior $(18,4 \mathrm{~cm})$ a do grupo ativo $(22,3 \mathrm{~cm})$. Embora se observe uma tendência de o grupo de idosas envolvido na prática de atividades físicas em apresentar um maior grau de flexibilidade em relação ao grupo que não as praticava, esta diferença não foi estatisticamente significativa $(p \geq 0,05)$.

\section{DISCUSSÃO}

Conforme os resultados apresentados na Tabela 1, verifica-se que o índice médio de massa corporal total da amostra apontou para um grupo classificado como obeso. Quando separada a amostra pela participação e não em atividades físicas, o grupo que praticava tais atividades apresentou um IMC beirando o padrão de obesidade $\left(27,0 \mathrm{~kg} / \mathrm{m}^{2}\right)$, já que a faixa da normalidade, segundo os pontos de corte de Lipschitz $^{10}$ é de 22 a $27 \mathrm{~kg} / \mathrm{m}^{2}$. O grupo não ativo classificou-se como obeso $\left(32,6 \mathrm{~kg} / \mathrm{m}^{2}\right)$.

A tabela 2 denota que o grupo não ativo fisicamente apresentou risco muito elevado para doenças cardiovasculares por meio da medição da circunferência da cintura. Esta propensão foi, também, observada através da relação cintura-quadril.

Matsudo $^{15}$ afirma que o ganho de peso e o acúmulo da gordura corporal são resultados de um padrão prolongado geneticamente de mudanças na dieta e no nível da atividade física. Com as alterações antropométricas, o aumento da gordura nas primeiras décadas do envelhecimento e a perda de gordura nas décadas mais tardias da vida parece ser o padrão mais provável de comportamento da adiposidade corporal com o processo de envelhecimento.
Segundo Nahas, em muitos casos, o declínio na atividade física habitual tem provocado um desequilíbrio no balanço energético que favorece o aumento de pessoas obesas, mesmo quando se observa um controle de calorias na alimentação diária. A redução do peso corporal é observada pela prática da atividade física, já que possibilita uma perda mais significativa de gordura corporal, preservação da massa muscular, controle do peso corporal, que proporcionará uma vida mais saudável.

Berlezi et al., ${ }^{16}$ em um estudo cujos resultados se assemelham aos da presente pesquisa, avaliaram 20 idosas acima de 60 anos, no município de Ijuí, RS, das quais, dez eram praticantes e dez não praticantes de atividades físicas. Foi, então, constatado que $60 \%$ das idosas não praticantes de atividades físicas apresentavam sobrepeso e 50\% delas risco muito alto para o desenvolvimento de doenças cardiovasculares. Dentre as idosas do grupo ativo fisicamente, $50 \%$ delas se encontravam na faixa normal para o IMC e $40 \%$ apresentavam risco moderado para a aquisição de tais doenças.

Mazo et al. ${ }^{17}$ realizaram um estudo para verificar a relação entre o Índice de Aptidão Funcional Geral (IAFG) e o IMC de mulheres idosas, praticantes de atividades físicas, em que constataram diferença estatisticamente significativa entre o IMC e o desempenho no IAFG, teste de capacidade aeróbia $(p=0,001)$. Foi, então, concluído que altos índices de IMC estavam associados a escores baixos no IAFG. Com o teste de capacidade aeróbia, observou-se uma tendência de 42,9\% das idosas, com IAFG bom, terem IMC normal e de $95,6 \%$ daquelas com IAFG fraco terem IMC de sobrepeso. Verificouse que as idosas com sobrepeso apresentaram um risco aproximadamente 16 vezes maior para terem um IAFG fraco em relação àquelas com IMC normal. Os resultados permitiram concluir que parece existir uma relação inversa entre o IMC e o IAFG de idosas, de forma que aquelas com sobrepeso têm grande risco de terem um IAFG fraco. 
Observa-se na tabela 2 que a frequência cardíaca final do grupo de idosas não ativas foi inferior ao do grupo das ativas fisicamente, embora não se tenha observado uma diferença estatisticamente significativa entre as médias desses grupos. Tal resultado foi surpreendente pelo fato de que se esperava um resultado superior no grupo ativo fisicamente, isto é, uma freqüência cardíaca final inferior a do grupo não ativo fisicamente.

Buscando-se dados adicionais na amostra do estudo, antes do início do teste, foi detectado que $65 \%$ das idosas participantes do grupo não ativo tomava medicamento para a hipertensão e apenas $45 \%$ das participantes do grupo ativo encontrava-se na mesma condição. De modo geral, os medicamentos betabloqueadores que fazem o bloqueio beta cardíaco, resultam em diminuição da condução atrioventricular e supressão da automação, redução de frequência e débitos cardíacos, com diminuição do trabalho do coração e do consumo miocárdico de oxigênio. Assim, acredita-se que o medicamento possa ter influenciado os resultados nesta variável.

Quando se considerou a distância percorrida em metros, observou-se, na tabela 2, que as participantes do grupo ativo percorreram uma distância média $(434,9 \mathrm{~m})$ superior a do grupo não ativo (383,3m). Farias, Mendivil \& GuerraJúnior, ${ }^{18}$ também, apresentaram resultados semelhantes em uma pesquisa realizada para avaliar a resistência aeróbia de mulheres adultas acima de 40 anos e mulheres idosas. Após cinco meses em um programa de caminhada orientada, foi constatado no teste do caminhar durante 6 minutos a contribuição do programa para a melhoria da aptidão física das participantes. Os resultados deste estudo reforçam a importância da atividade física rotineira e orientada para a melhora das condições de saúde e de vida. Os autores salientam, entretanto, que os efeitos de um programa de treinamento aeróbio em grupos de pessoas de meia-idade e idosos sobre o fortalecimento da musculatura são rapidamente perdidos com a sua suspensão. Este fato direciona para a necessidade de estudos que proporcionem o entendimento de aspectos relacionados à aderência e à permanência de pessoas adultas em programas de atividade física.

Comparando-se os resultados da presente investigação, foi detectada semelhança com os resultados do estudo conduzido por Furtado et al. ${ }^{19}$ que constataram a melhoria do perfil da resistência cardiorrespiratória de idosos ativos nele participantes. No teste de 6 minutos, foi observado que o grupo de idosas de 60 a 69 anos apresentou melhores resultados neste teste: G1(60 a 64 anos) $=526 \mathrm{~m}$ e G2(65 a 69 anos) $=509,7 \mathrm{~m}$. Nos grupos compostos por idosas de 70 a 79 anos os resultados foram inferiores: G3(70 a 74 anos) $=491,3 \mathrm{~m}$ e G4(75 a 79 anos) $=479,4 \mathrm{~m}$. Considerando a possibilidade da não especificidade da prescrição do exercício físico para cada faixa etária apresentada pelos grupos estudados, o programa de atividade física realizado foi capaz de proporcionar a $60,5 \%$ daquelas idosas, um perfil satisfatório para a resistência cardiorrespiratória.

Rikli \& Jones ${ }^{12}$ postulam que a preservação de níveis adequados de resistência aeróbia é importante para a qualidade de vida do idoso, já que possibilita a prevenção de incapacidades ocasionadas pelo processo de envelhecimento, como também, facilita a manutenção das atividades básicas e instrumentais de vida diária, como: realizar a higiene pessoal; alimentar-se; vestir-se; locomover-se pela casa de forma segura e independente; ir às compras, ao banco, visitar amigos, cozinhar, entre outras atividades.

Para Nieman ${ }^{6}$ entre os 30 e 70 anos de idade, o volume de força diminui em média 30 por cento, geralmente pela falta de prática de atividades físicas, o que contribui para a fraqueza e fragilidade, comum nos idosos. Pessoas idosas que se exercitam com pesos recuperam uma boa parte de sua força perdida, o que as capacita para um melhor desempenho das atividades diárias.

Pereira et al. $^{20}$ ao avaliar a força de membros superiores e inferiores de 540 idosas divididas em grupo ativo fisicamente $(\mathrm{n}=358)$ e sedentário $(\mathrm{n}=182)$ concluíram que no que se refere aos membros superiores, os dois grupos se encontravam dentro dos índices preditos. 
Entretanto, o índice relativo à força de membros inferiores foi inferior no grupo sedentário quando comparado ao ativo fisicamente que se classificou dentro dos valores mínimos preditos recomendados. Isto representa uma vulnerabilidade dos níveis de força funcional de membros inferiores dos grupos. Para os autores, essa condição pode ter ocorrido pela possível ausência de uma sessão específica de treinamento de força nos programas de atividade física em que as idosas participavam e pelo fato de o sedentarismo ser uma variável importante no processo de diminuição da força, com efeito, mais contundente no sexo feminino.

Observa-se, então, certa semelhança dos resultados do estudo de Pereira et al. ${ }^{20}$ aos dados apresentados na tabela 2 quando o grupo ativo apresentou resultados superiores aos do não ativo fisicamente neste teste.

A tabela 2 informa que o grupo de idosas ativas fisicamente apresentou uma flexibilidade superior $(22,3 \mathrm{~cm})$ ao do grupo não ativo $(18,4$ $\mathrm{cm})$. Assim, se tais resultados são comparados com os que a literatura apresenta, comprova-se que a razão para estes resultados é o fato de ser documentado que a flexibilidade apresenta relação com a idade e com a atividade física. Conforme a pessoa envelhece, a flexibilidade diminui, embora se acredite que a sua causa principal seja a falta de atividade física. ${ }^{6}$ A melhoria da amplitude articular pode ser melhorada, segundo Dantas, ${ }^{21}$ através de um programa de atividade física adequado às necessidades do idoso.

Os dados do teste de flexibilidade do presente estudo corroboram, também, os apresentados por Silva \& Rabelo ${ }^{22}$ que evidenciaram uma melhoria no nível de flexibilidade em idosas que participavam de atividades físicas $(30 \mathrm{~cm})$ quando comparado ao do grupo que não as praticavam $(22,7 \mathrm{~cm})$. Os autores, com base nestes resultados, puderam observar a possível contribuição da prática da atividade física para a melhoria dos níveis de flexibilidade da amostra de seu estudo.

Berlezi et al. ${ }^{16}$ ao testar a flexibilidade em idosas constataram um índice superior no grupo que participava em atividades físicas quando comparado ao do grupo não ativo. $\mathrm{O}$ resultado obtido pelo grupo ativo sugere que a flexibilidade pode ser positivamente influenciada com a prática de atividade física. Observa-se, então, pelos estudos apresentados nesta discussão sobre a flexibilidade, certa uniformidade nos resultados relatados, o que demonstra a influência da prática da atividade física na melhoria dos níveis de flexibilidade de idosos.

\section{CONCLUSÃO}

Embora não se tenham constatado resultados significativos entre os grupos de idosas estudados no teste de capacidade cardiorrespiratória, nos demais testes, os resultados foram favoráveis ao grupo que praticava atividade física três vezes por semana. Tal fato denota a importância da prática regular da atividade física na melhoria da aptidão física relacionada à saúde. Recomenda-se, em futuros estudos, um maior controle de variáveis que possam influenciar os resultados em testes cardiorrespiratórios, tais como: medicamentos betabloqueadores.

\section{REFERÊNCIAS}

1. Brasil. Ministério do Planejamento, Orçamento e Gestão. Instituto Brasileiro de Geografia e Estatística.Síntese de Indicadores Sociais: uma análise das condições de vida da população brasileira. Rio de Janeiro: IBGE, 2010 [Acesso em: 23 mar. 2011]. Disponível em: http://www1.ibge.gov.br/ home/estatistica/populacao/condicaodevida/ indicadoresminimos/sinteseindicsociais2010/ SIS_2010.pdf.

2. Okuma SS. O idoso e a atividade física: fundamentos e pesquisas. Campinas: Papirus; 1998.

3. Spirduso WW. Dimensões físicas do envelhecimento. Trad. Paula Bernardi. Barueri [SP]: Manole; 2005. Título original: Physical dimensions of ageing.

4. World Health Organization. Envelhecimento ativo: uma política de saúde. Trad. Suzana Gontijo. Brasília: Organização Pan-Americana da Saúde; 2005. 
5. Lacourt MX, Marini LL. Decréscimo da função muscular decorrente do envelhecimento e a influência da qualidade de vida do idoso: uma revisão de literatura. Revista Brasileira de Ciências do Envelhecimento Humano. 2006; 3(1):114-121.

6. Nieman DC. Exercício físico e saúde. São Paulo: Manole; 1999.

7. Nahas MV. Atividade física, saúde e qualidade de vida: conceitos e sugestões para um estilo de vida ativo. 3. ed. Londrina: Midiograf; 2003.

8. Pitanga FJG. Epidemiologia da atividade física, exercício físico e saúde. 2.ed. São Paulo: Phorte; 2004.

9. Brasil. Ministério do Planejamento, Orçamento e Gestão. Instituto Brasileiro de Geografia e Estatística. IBGE Cidades@: sinopse do Censo Demográfico 2010. Rio de Janeiro: IBGE; 2010b. [Acesso em: 1 jul. 2011]. Disponível em: http://www. ibge.gov.br/cidadesat/topwindow.htm?1

10. Lipschitz DA. Screening for nutritional status in the elderly. Primary Care 1994; 21(1):55-67.

11. Word Health Organization. Obesity. Preventing and managing the global epidemic. Geneva: Report of a WHO Consultation on Obesity. Geneva: WHO; 1998.

12. Rikli RE, Jones J. Teste de aptidão física para idosos. São Paulo: Manole; 2008.

13. Pollock ML, Wilmore JH, Fox SM. Exercício na saúde e na doença: avaliação e prescrição para prevenção e reabilitação. 2. ed. Rio de Janeiro: Médica e Científica; 1993.

14. Matsudo SM. Avaliação da Aptidão Física. In: Matsudo SMM. Avaliação do idoso: física e funcional. 2. ed. Londrina: Midiograf; 2004.

15. Matsudo SM, Matsudo VKR, Barros Neto TL. Atividade física e envelhecimento: aspectos epidemiológicos. Rev Bras Med Esporte 2001; 7(1):2-13.

16. Berlezi EM, Rosa PV, Souza ACA, Schneider RH. Comparação antropométrica e do nível de aptidão física de mulheres acima de 60 anos praticantes de atividade física regular e não praticantes. Rev. Bras.
Geriatr. Gerontol. [periódico na Internet]. 2006 [citado 2011 Jul 01]; 9(3):49-66. Disponível em: http://revista.unati.uerj.br/scielo.php?script $=$ sci_ arttext\&pid=S1809-98232006000300005\&lng=pt.

17. Mazo GZ, Külkamp W, Lyra VB, Prado APM. Aptidão funcional geral e índice de massa corporal de idosas praticantes de atividade física. Rev. Bras. Cineantropom. Desempenho Hum 2006; 8(4):46-51.

18. Farias ES, Mendivil FHS, Guerra-Junior G. Efeitos do treinamento aeróbio sobre componentes da aptidão física relacionada à saúde em mulheres adultas. R. da Educação Física/UEM 2008; 19(4):591-598.

19. Furtado HL, Pereira FD, Moreira MHR, Dantas EHM. Perfil da resistência cardiorrespiratória em mulheres idosas com sobrepeso do programa de atividade física no SESC de Nova Friburgo, RJ, Brasil. Revista de Desporto e Saúde 2007; 4(1):21-26.

20. Pereira FD, Batista WO, Furtado HL, Alves Junior ED, Giani TS, Dantas EHM. Comparação da força funcional de membros inferiores e superiores entre idosas fisicamente ativas e sedentárias. Rev. Bras. Geriatr. Gerontol. [periódico na Internet]. 2009 [citado 2011 Jul 01];12(3):417-428. Disponível em: http://revista.unati.uerj.br/scielo.php?script=sci_ arttext\&pid=S1809-98232009000300009\&lng=pt.

21. Dantas EHM. Flexibilidade, alongamento e flexionamento. 4. ed. Rio de Janeiro: Shape; 1999.

22. Silva M, Rabelo HT. Estudo comparativo dos níveis de flexibilidade entre mulheres idosas praticantes de atividade física e não praticantes. MOVIMENTUM - Revista Digital de Educação Física [periódico na Internet]. 2006 ago [citado 2011 Jul 01];1. Disponível em: http://www.unilestemg. br/movimentum/Artigos_V1N1_em_pdf/ movimentum_silva_margareth.pdf 